\title{
UTILIZAÇÃO DE LÂMINAS DE Eucalyptus grandis e Eucalyptus maculata COMO ALTERNATIVAS PARA PRODUÇÃO DE PISO LAMINADO COM MONTAGEM DIRETA
}

\author{
Setsuo Iwakiri*, Andréa Berriel Mercadante Stinghen**, Elenise Leocádia da Silveira Nunes**, \\ Esoline Helena Cavalli Zamarian***, Márcia Keiko Ono Adriazola** \\ *Eng. Florestal, Dr., Depto. de Engenharia e Tecnologia Florestal, UFPR - setsuo@ufpr.br \\ **Doutoranda em Engenharia Florestal, UFPR \\ ***Mestranda em Engenharia Florestal, UFPR \\ Recebido para publicação: 08/06/2006 - Aceito para publicação: 16/08/2006
}

\begin{abstract}
Resumo
Este trabalho foi desenvolvido com o objetivo de avaliar a qualidade dos painéis compensados do tipo "combi", produzidos com lâminas de Eucalyptus grandis e Eucalyptus maculata como capa e contracapa, e lâminas de Pinus taeda como miolo, visando sua aplicação como piso laminado com montagem direta. Os painéis foram produzidos com resina fenol-formaldeído e melamina-uréiaformaldeído. As avaliações do coeficiente de redução em espessura, resistência da linha de cola, flexão estática e dureza Janka demonstraram que os painéis produzidos com capa e contracapa de $E$. maculata e miolo com $P$. taeda, com uso tanto de resina fenol-formaldeído quanto de melaminauréia-formaldeído, foram os que apresentaram melhores propriedades para uso como piso laminado de madeira. Os resultados indicam ainda a possibilidade de utilização do processo de "montagem direta" para fabricação de pisos laminados.

Palavras-chave: Compensado; piso laminado; lâminas de madeira.
\end{abstract}

\begin{abstract}
Utilization of veneer of Eucalyptus grandis and Eucalyptus maculata as the alternatives to manufactures laminated wood flooring trough direct assembly method. This research was developed aiming to evaluate the quality of plywood manufactured using veneer of Eucalyptus grandis and Eucalyptus maculata as face layer and Pinus taeda as core, to use for laminated wood flooring. The boards were manufactured with phenol-formaldehyde and melamine-urea-formaldehyde resins. The evaluation of the coefficient of thickness reduction, glue line strength, bending strength and surface hardness, showed that the boards made from E. maculata as face layer and P. taeda as core, using with phenol-formaldehyde and melamine-urea-formaldehyde resins, presented the better properties to use as laminated wood flooring. The results also showing the possibility to use the direct assembly method to laminated flooring manufacture.

Keywords: Plywood; laminated wood flooring; veneer.
\end{abstract}

\section{INTRODUÇÃO}

A produção de pisos de madeira no Brasil vem crescendo expressivamente a partir de meados da década de 90, tendo alcançado em 2004 cerca de 24 milhões de $\mathrm{m}^{2}$, sendo 18 milhões de $\mathrm{m}^{2}(75 \%)$ de pisos "engenheirados" e 6 milhões de $\mathrm{m}^{2}(25 \%)$ de pisos maciços. Esse aumento pode ser atribuído principalmente ao crescimento da produção de pisos "engenheirados", que passaram a ser difundidos no mercado e amplamente empregados na construção civil. Por sua vez, o piso maciço é produzido normalmente com madeiras nobres e mantém-se ainda com destaque, porém, com produção menor e sem crescimento significativo nos últimos dez anos (ABIMCI, 2005).

O termo "piso engenheirado" é de uso recente, o qual é um produto fabricado a partir de painéis compensados ou painéis de fibras de alta densidade (HDF - high density fiberboard) revestidos com lâminas decorativas de madeira ou papéis melamínicos. No processo de fabricação de pisos "engenheirados" (laminados), são utilizadas lâminas de madeira coladas como em painel compensado, 
formando a base do piso. Na fase seguinte, o compensado passa por processo de calibração e lixamento, para ajuste da espessura, e recebe a camada de lâminas de madeira decorativa na face. A fase final consiste de cortes longitudinais em larguras finais do piso, perfilamento longitudinal e transversal para encaixes e aplicação de material de acabamento superficial.

Os pisos de madeira, tanto maciços quanto engenheirados, são produtos provenientes de recursos naturais renováveis. Nesse contexto, Baldwin (1995) cita uma comparação entre pisos de madeira e "carpet", enfocando a energia líquida (megawatts) necessária para a produção de uma tonelada desses dois produtos. Segundo o autor, é necessário 0,85 megawatts para produção de uma tonelada de pisos de madeira, contra 3,59 megawatts para uma tonelada de carpet, produzido a partir de material sintético e não renovável. Steida (1992) destaca também as vantagens em termos de custo-benefício dos pisos de madeira em relação aos outros materiais, principalmente quanto à beleza, variedade de estilos e cores das diferentes espécies de madeira.

Por outro lado, os pisos "engenheirados", por serem produtos colados de madeira, apresentam uma série de vantagens em relação aos pisos de madeira maciça. A primeira refere-se ao melhor aproveitamento da madeira no processo de obtenção de lâminas, sem a geração de resíduos, tanto na forma de serragens no processamento primário quanto na forma de cepilhos no processo de remanufatura. A segunda, refere-se à maior estabilidade dimensional das peças. O princípio de laminação cruzada, aliada à restrição imposta pela linha de cola às diferentes alterações dimensionais das camadas adjacentes, resulta em produto com maior estabilidade dimensional, quando comparado à madeira maciça sob influência do fator anisotrópico (TSOUMIS, 1993; SELLERS, 1992).

As principais espécies utilizadas na produção de pisos engenheirados (laminados) no Brasil são: amescla (Trattinickia burserifolia), assacu (Hura crepitans), faveira (Parkia spp.), caju-açu, cajuí (Anacardium spp.), ucuúba (Virola spp.), entre outras, para "miolão" ou base do piso, e lâminas faqueadas de marfim (Balfourodendrum riedelianum), ipê (Tabebuia spp.), angelim (Hymenolobium spp.), sucupira (Bowdichia nitida), muiracatiara (Astronium spp.), entre outras, para capa (Revista da Madeira, 2005).

De acordo com Castro (2002), a matéria-prima básica, tradicionalmente oriunda de florestas, está sendo gradativamente substituída por produtos sólidos e reconstituídos, provenientes de plantios de espécies arbóreas de rápido crescimento, cujas espécies utilizadas apresentam, entre outras vantagens, alta produtividade, redução da idade de corte, segurança de abastecimento, homogeneidade de matéria-prima, custo competitivo, produção regionalizada, além da possibilidade de múltiplos usos da floresta e seus produtos.

No Brasil, algumas espécies de eucaliptos provenientes de plantios podem ser alternativas promissoras para a produção de pisos "engenheirados", em substituição às espécies tropicais provenientes de floresta da região amazônica. De acordo com Hillis (2000), entre as espécies do gênero Eucalyptus e a do gênero Pinus, a madeira de eucalipto apresenta consideráveis vantagens sobre a madeira de pinus (coníferas), devido à melhor aparência, maior resistência e rigidez para usos estruturais, além da extrema plasticidade do gênero.

Este trabalho teve como objetivo avaliar as propriedades físicas e mecânicas dos compensados do tipo "combi", produzidos com lâminas de Eucalyptus grandis e Eucalyptus maculata como capa e contracapa e Pinus taeda como miolo, coladas com resina fenol-formaldeído e melamina-uréiaformaldeído, visando a sua utilização como pisos laminados com montagem direta.

\section{MATERIAIS E MÉTODOS}

Foram utilizadas, nesta pesquisa, lâminas de Pinus taeda, Eucalyptus grandis e Eucalyptus maculata, com espessura nominal de 2,0 mm. As lâminas de Pinus taeda e Eucalyptus grandis foram obtidas diretamente de indústrias laminadoras localizadas na região de Telêmaco Borba (PR), e as de Eucalyptus maculata foram obtidas a partir de árvores com trinta anos, coletadas na Floresta Estadual de Santa Bárbara do Rio Pardo (SP), pertencente ao Instituto Florestal do Estado de São Paulo, tendo sido laminadas numa indústria em Curitiba (PR). As resinas empregadas para a colagem das lâminas foram a fenol-formaldeído e a melamina-uréia-formaldeído, com teor de sólidos de $49 \%$ e $64 \%$, respectivamente.

As lâminas foram secadas ao teor de umidade médio de $6 \%$, em estufa de laboratório com circulação forçada de ar. Antes da aplicação da cola, as lâminas, em número de quatro, foram separadas e 
identificadas de acordo com a composição de cada painel compensado dos respectivos tratamentos, sendo medidas as espessuras em quatro pontos da lâmina. A somatória da espessura das quatro lâminas foi utilizada para posterior avaliação da redução em espessura do painel.

Os painéis foram montados com quatro lâminas, sendo as duas internas em camada unidirecional, perpendicular às lâminas da capa e contracapa. Foram produzidos um total 18 painéis com dimensões de 50 x $50 \mathrm{~cm}$, sendo 3 repetições para cada um dos 6 tratamentos, conforme delineamento experimental apresentado na tabela 1 .

Tabela 1. Delineamento experimental.

Table 1. Experimental chart.

\begin{tabular}{lcc}
\hline Tratamento & $\begin{array}{c}\text { Composição do painel } \\
\text { capa / miolo / contracapa }\end{array}$ & Tipo de resina \\
\hline T1 & P.taeda / P. taeda / P. taeda & Fenol-formaldeído \\
T2 & E. grandis / P. taeda / E. grandis & Fenol-formaldeído \\
T3 & E. maculata / P. taeda / E. maculata & Fenol-formaldeído \\
T4 & E. grandis / E. grandis / E. grandis & Fenol-formaldeído \\
T5 & E. grandis / P. taeda / E. grandis & Melamina-uréia-formaldeído \\
T6 & E. maculata / P. taeda / E. maculata & Melamina-uréia-formaldeído \\
\hline
\end{tabular}

Para a colagem de lâminas, foi utilizada gramatura (linha simples) de $180 \mathrm{~g} / \mathrm{m}^{2}$ para resina fenolformaldeído e de $175 \mathrm{~g} / \mathrm{m}^{2}$ para resina melamina-uréia-formaldeído. Os painéis foram prensados nas seguintes condições: (1) resina fenol-formaldeído: temperatura $=140{ }^{\circ} \mathrm{C}$, tempo de prensagem $=8$ minutos, pressão específica $=10 \mathrm{kgf} / \mathrm{cm}^{2}$ (T1) e $15 \mathrm{kgf} / \mathrm{cm}^{2}$ (T2 a T6); (2) resina melamina-uréiaformaldeído: temperatura $=100{ }^{\circ} \mathrm{C}$, tempo de prensagem $=7$ minutos, pressão específica $=10 \mathrm{kgf} / \mathrm{cm}^{2}$ (T1) e $15 \mathrm{kgf} / \mathrm{cm}^{2}$ (T2 a T6).

Após a prensagem e estabilização dos painéis na sala de climatização à temperatura de $20 \pm 1{ }^{\circ} \mathrm{C}$ e umidade relativa de $65 \pm 3 \%$, até atingir o teor de umidade médio de $12 \%$, foram tomadas as medidas de espessuras em quatro pontos do painel, para avaliação da redução média em espessura devido à compressão da madeira na fase de prensagem à quente. A redução em espessura do painel foi determinada pela subtração da soma das espessuras das lâminas pela espessura do painel correspondente. Adicionalmente, foi determinado o coeficiente de redução em espessura do painel acondicionado em relação à somatória da espessura das lâminas correspondentes.

Para a avaliação das propriedades mecânicas dos painéis, foram retirados os corpos de prova para realização dos seguintes ensaios: resistência da linha de cola aos esforços de cisalhamento (teste seco e úmido), flexão estática paralela para determinação do módulo de elasticidade e módulo de ruptura, e dureza Janka. Os procedimentos para os ensaios foram de acordo com a norma EM 314:1993 para resistência da linha de cola e EN 310:1993 para flexão estática. Para a dureza Janka foi utilizada a norma ASTM D 143-94 (1996) para madeira sólida.

Os resultados obtidos nos ensaios foram analisados através de delineamento inteiramente casualizado, com aplicação da análise de variância e teste de Tukey ao nível de probabilidade de 95\%, para comparação das médias entre os tratamentos.

\section{RESULTADOS E DISCUSSÃO}

As reduções em espessuras dos painéis compensados constituídos de lâminas de $P$. taeda, E. grandis e E. maculata, com diferentes composições, estão apresentados na tabela 2 e ilustradas no gráfico da figura 1. Os resultados demonstram uma redução acentuada na espessura dos painéis produzidos com 100\% de lâminas de Pinus, em comparação aos painéis tipo "combi", produzidos com lâminas de $E$. grandis e E. maculata nas capas e contracapas, e miolo com lâminas de Pinus. Essa diferença pode ser atribuída à menor densidade da madeira de Pinus em comparação à madeira de E. grandis e E. maculata, e, conseqüentemente, à menor resistência oferecida à compressão durante o processo de prensagem à alta temperatura. Cabe ressaltar ainda que a madeira de E. grandis e E. maculata, com maior densidade, além de ter maior resistência à compressão, possui maior força de expansão para retorno em espessura durante 
o processo de estabilização dos painéis na câmara de climatização. Esse resultado é de grande importância para a produção de piso laminado com "montagem direta", tendo em vista possibilitar o ajuste da espessura final do produto e o acabamento superficial em única etapa de calibração e lixamento. O processo de "montagem direta" elimina a etapa de colagem de lâminas decorativas, posterior às etapas de montagem da base do piso laminado (miolão), calibração e lixamento do painel compensado.

Tabela 2. Redução em espessura dos painéis compensados.

Table 2. Thickness reduction in the plywood.

\begin{tabular}{lcccc}
\hline Composição do painel & EL $(\mathbf{m m})$ & EP $(\mathbf{m m})$ & RE $(\mathbf{m m})$ & CR $\mathbf{( \% )}$ \\
\hline Pinus & 10,00 & 8,80 & 1,20 & $-12,00$ \\
E. grandis / Pinus & 8,51 & 8,20 & 0,31 & $-3,64$ \\
E. maculata / Pinus & 9,11 & 8,74 & 0,37 & $-4,06$ \\
\hline
\end{tabular}

EL: somatória da espessura das lâminas; EP: espessura do painel; RE: redução em espessura; CR: coeficiente de redução em espessura.

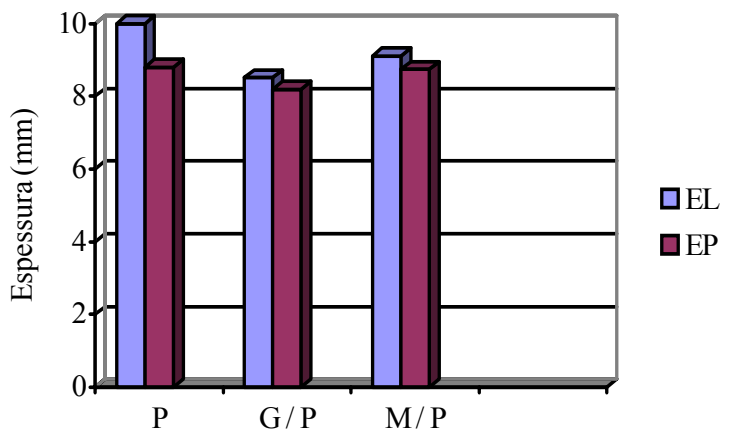

Figura 1. Representação gráfica da redução em espessura dos painéis compensados (onde: P: Pinus taeda; G: E. grandis; M: E. maculata; EL: somatória das espessuras das lâminas do painel (mm); EP: espessura do painel (mm)).

Figure 1. Representative chart of thickness reduction in the plywood.

Os valores médios referentes à resistência da linha de cola aos esforços de cisalhamento estão apresentados na tabela 3. Para o teste seco, verifica-se que os painéis produzidos integralmente com lâminas de E. grandis apresentaram valor médio de resistência da linha de cola estatisticamente inferior em relação aos painéis produzidos com lâminas de Pinus no miolo. Essa diferença indica que as interações entre as madeiras de Eucalyptus com a de Pinus resultam em melhores condições de colagem. Ou seja, a maior porosidade da madeira de Pinus gera condições mais favoráveis para formação da ligação adesiva entre as duas lâminas. Situação oposta foi constatada nas interações entre as lâminas de Eucalyptus (T4), com menor porosidade da madeira em função da sua maior densidade. Nessas condições, as ações de mobilidade do adesivo, relacionadas à penetração e umectação, não foram satisfatórias, resultando em menor resistência da ligação adesiva.

Nos ensaios realizados em corpos de prova após imersão em água por período de 24 horas (teste úmido), não foram constatadas diferenças estatisticamente significativas entre os valores médios obtidos para todos os tratamentos. Os valores médios de resistência da linha de cola no teste úmido, obtidos para todos os tratamentos, estão acima do valor mínimo de $1.0 \mathrm{Mpa}\left(10,197 \mathrm{kgf} / \mathrm{cm}^{2}\right)$, estabelecido pela norma EN 314:1993, para compensado fenólico destinado ao uso exterior. Esse resultado atende a um dos requisitos importantes para pisos laminados de madeira. Quanto aos diferentes tipos de resinas, não foram constatadas diferenças estatisticamente significativas entre os painéis produzidos com resina fenolformaldeído e melamina-uréia-formaldeído, como mostram as interações entre os tratamentos T2 x T5 e T3 x T6. 
Tabela 3. Valores médios dos ensaios de resistência da linha de cola.

Table 3. Average values of glue line strength.

\begin{tabular}{lcc}
\hline Tratamento & RLC - teste seco $\left(\mathbf{k g f} / \mathbf{c m}^{\mathbf{2}}\right)$ & RLC - teste úmido $\left(\mathbf{k g f} / \mathbf{c m}^{\mathbf{2}}\right)$ \\
\hline $\mathrm{T} 1-\mathrm{Pt} / \mathrm{Pt} / \mathrm{Pt}-\mathrm{ff}$ & $22,79 \mathrm{~b}$ & $12,62 \mathrm{a}$ \\
$\mathrm{T} 2-\mathrm{Eg} / \mathrm{Pt} / \mathrm{Eg}-\mathrm{ff}$ & $20,54 \mathrm{~b}$ & $13,20 \mathrm{a}$ \\
$\mathrm{T} 3-\mathrm{Em} / \mathrm{Pt} / \mathrm{Em}-\mathrm{ff}$ & $23,23 \mathrm{~b}$ & $12,82 \mathrm{a}$ \\
$\mathrm{T} 4-\mathrm{Eg} / \mathrm{Eg} / \mathrm{Eg}-\mathrm{ff}$ & $12,20 \mathrm{a}$ & $11,68 \mathrm{a}$ \\
$\mathrm{T} 5-\mathrm{Eg} / \mathrm{Pt} / \mathrm{Eg}-$ muf & $18,82 \mathrm{~b}$ & $14,92 \mathrm{a}$ \\
$\mathrm{T} 6-\mathrm{Em} / \mathrm{Pt} / \mathrm{Em}-$ muf & $20,97 \mathrm{~b}$ & $15,10 \mathrm{a}$ \\
\hline
\end{tabular}

Médias seguidas de mesma letra são estatisticamente iguais ao nível de probabilidade de 95\%. Pt: Pinus taeda; Eg: Eucalyptus grandis; Em: Eucalyptus maculata; ff: resina fenol-formaldeído; muf: resina melamina-uréia-formaldeído; RLC: resistência da linha de cola.

Com relação aos resultados das propriedades de flexão estática e dureza Janka apresentados na tabela 4, pode-se constatar que os painéis produzidos com lâminas de E. maculata nas capas e $P$. taeda no miolo, correspondente ao tratamento T3, apresentaram valores de módulo de elasticidade e módulo de ruptura superiores em comparação aos demais tratamentos. Para o módulo de ruptura, essa diferença foi estatisticamente significativa. Como referência, no trabalho realizado por Interamnense (1998) para painéis compensados de E. maculata, foram obtidos valores médios para MOE e MOR de 160.328 $\mathrm{kgf} / \mathrm{cm}^{2}$ e $706 \mathrm{kgf} / \mathrm{cm}^{2}$, respectivamente. Esses valores estão próximos ao valor médio do MOE de $156.172 \mathrm{kgf} / \mathrm{cm}^{2}$ e muito inferior ao valor médio de MOR de 1.005,67 kgf $/ \mathrm{cm}^{2}$, obtidos neste estudo para o tratamento T3. A composição E. maculata-P. taeda resultou também em maiores valores médios de dureza Janka. A utilização da madeira de E. maculata, com maior densidade, na capa e contracapa do painel, contribuíram para os aumentos nos valores médios das propriedades de flexão estática e dureza Janka dos painéis. Esse resultado é muito importante sob o ponto de vista de composição do painel compensado, visando otimização do balanço estrutural do painel em termos de resistência mecânica.

Tabela 4. Valores médios dos ensaios de flexão estática e dureza Janka.

Table 4. Average values of static bending and Janka hardness.

\begin{tabular}{lccc}
\hline Tratamento & MOE $\mathbf{( k g f / \mathbf { c m } ^ { 2 } )}$ & MOR $\mathbf{( k g f / \mathbf { c m } ^ { 2 } )}$ & Dureza Janka $(\mathbf{k g f})$ \\
\hline $\mathrm{T} 1-\mathrm{Pt} / \mathrm{Pt} / \mathrm{Pt}-\mathrm{ff}$ & $121.144 \mathrm{a}$ & $780 \mathrm{a}$ & $539,17 \mathrm{a}$ \\
$\mathrm{T} 2-\mathrm{Eg} / \mathrm{Pt} / \mathrm{Eg}-\mathrm{ff}$ & $130.874 \mathrm{a} \mathrm{b}$ & $934 \mathrm{a}$ & $626,50 \mathrm{a}$ \\
$\mathrm{T} 3-\mathrm{Em} / \mathrm{Pt} / \mathrm{Em}-\mathrm{ff}$ & $156.172 \mathrm{~b}$ & $1.234 \mathrm{~b}$ & $1.005,67 \mathrm{~b}$ \\
$\mathrm{~T} 4-\mathrm{Eg} / \mathrm{Eg} / \mathrm{Eg}-\mathrm{ff}$ & $134.085 \mathrm{a} \mathrm{b}$ & $865 \mathrm{a}$ & $722,17 \mathrm{a} \mathrm{b}$ \\
$\mathrm{T} 5-\mathrm{Eg} / \mathrm{Pt} / \mathrm{Eg}-$ muf & $123.737 \mathrm{a}$ & $793 \mathrm{a}$ & $450,17 \mathrm{a}$ \\
$\mathrm{T} 6-\mathrm{Em} / \mathrm{Pt} / \mathrm{Em}-$ muf & $133.719 \mathrm{a} \mathrm{b}$ & $955 \mathrm{a}$ & $981,67 \mathrm{~b}$
\end{tabular}

Médias seguidas de mesma letra são estatisticamente iguais ao nível de probabilidade de 95\%. Pt: Pinus taeda; Eg: Eucalyptus grandis; Em: Eucalyptus maculata; ff: resina fenol-formaldeído; muf: resina melamina-uréia-formaldeído; MOE: módulo de elasticidade; MOR: módulo de ruptura.

\section{CONCLUSÕES} apresentadas:

Com base nos resultados obtidos nesta pesquisa, as seguintes conclusões podem ser

- Os coeficientes de redução em espessura dos painéis produzidos com lâminas de E. grandis e E. maculata nas capas e contracapas e lâminas de $P$. taeda no miolo foram menores em comparação aos painéis produzidos integralmente com lâminas de $P$. taeda. Esse resultado indica a possibilidade de produção de piso laminado de madeira através do processo de montagem direta, tendo em vista ocorrerem perdas menores em espessura na prensagem dos painéis, viabilizando dessa forma a calibração e o lixamento do piso laminado na espessura final.

- As interações entre as lâminas de P. taeda com lâminas de E. grandis e E. maculata resultaram em maior resistência da ligação adesiva, quando comparadas às interações entre as lâminas de $E$. grandis. 
- Os resultados obtidos nos ensaios de resistência da linha de cola, flexão estática e dureza Janka permitem afirmar que os painéis produzidos com lâminas de E. maculata na capa e contracapa e de Pinus no miolo apresentam propriedades satisfatórias como material estrutural, com possibilidades de utilização como piso laminado de madeira.

\section{REFERÊNCIAS}

AMERICAN SOCIETY FOR TESTING AND MATERIALS. ASTM D 143-94: methods of testing of small clear specimens. [S.1.], 1996.

ASSOCIAÇÃO BRASILEIRA DA INDÚSTRIA DA MADEIRA PROCESSADA MECANICAMENTE. Estudo Setorial 2004. Curitiba, 2005. 50p.

BALDWIN, R. F. Plywood and veneer-based products: manufacturing practices. San Francisco: Miller Freeman, 1995. 388p.

EUROPEAN COMMITTEE FOR STANDARDIZATION. EN 314-2: Plywood - Determination of shear bonding strength. [Brussels], 1993.

EUROPEAN COMMITTEE FOR STANDARDIZATION. EN 310: Plywood - Determination of bending strength. [Brussels], 1993.

HILLIS, W. E. Wood quality and growing to meet market requirements. In: THE FUTURE OF EUCALYPTUS FOR WOOD PRODUCTS, 2000, Launceston, Tasmania. Proceedings... Launceston: IUFRO, 2000. p.256-264.

INDUSTRIALIZAÇÃO viabiliza custo da madeira. Revista da Madeira, Curitiba, n.91, p.44-50, 2005.

INTERAMNENSE, M. T. Utilização das madeiras de Eucalyptus cloeziana (F. Muell), Eucalyptus maculata (Hook) e Eucalyptus punctata DC var. punctata para produção de painéis compensados. 81f. Dissertação (Mestrado em Ciências Florestais) - Setor de Ciências Agrárias, Universidade Federal do Paraná, Curitiba, 1998.

SELLERS, T. Plywood and adhesive technology. New York: Marcel Dekker, 1985. 661p.

SILVA, J. C. Caracterização da madeira de Eucalyptus grandis Hill ex. Maiden, de diferentes idades, visando a sua utilização na indústria moveleira. 160f. Tese (Doutorado em Ciências Florestais) - Setor de Ciências Agrárias, Universidade Federal do Paraná, Curitiba, 2002.

STEIDA, C. In: INTERNATIONAL CONFERENCE PROCEEDINGS. Wood product demand and the environment. [S.1.: s.n.], 1992. p.225.

TSOUMIS, G. Science and technology of wood: stucture, properties and utilization. New York: Van Nostrand Reinhold, 1991. 494p. 